\title{
Activation of the Extracellular Signal-Regulated Kinase in the Amygdala Modulates Pain Perception
}

\author{
Yarimar Carrasquillo ${ }^{1,2}$ and Robert W. Gereau IV ${ }^{1}$ \\ ${ }^{1}$ Washington University Pain Center and Department of Anesthesiology, Washington University School of Medicine, St. Louis, Missouri 63110, and \\ ${ }^{2}$ Department of Neuroscience, Baylor College of Medicine, Houston, Texas 77030
}

The amygdala has been proposed to serve as a neural center for the modulation of pain perception. Numerous anatomical and behavioral studies demonstrate that exogenous manipulations of the amygdala (i.e., lesions, drug infusions) modulate behavioral responses to acute noxious stimuli; however, little is known about the endogenous molecular changes in the amygdala that contribute to alterations in nociceptive processing during persistent noxious stimuli that resemble pathological pain conditions. In the present study, we demonstrate that endogenous molecular changes in the amygdala play a crucial role in modulating long-lasting peripheral hypersensitivity associated with persistent inflammation and we further identify the extracellular signal-regulated kinase (ERK) as a molecular substrate underlying this behavioral sensitization. Using the formalin test as a mouse model of persistent inflammatory pain, we show that activation of ERK in the amygdala is both necessary for and sufficient to induce long-lasting peripheral hypersensitivity to tactile stimulation. Thus, blockade of inflammation-induced ERK activation in the amygdala significantly reduced long-lasting peripheral hypersensitivity associated with persistent inflammation, and pharmacological activation of ERK in the amygdala induced peripheral hypersensitivity in the absence of inflammation. Importantly, blockade of ERK activation in the amygdala did not affect responses to acute noxious stimuli in the absence of inflammation, indicating that modulation of nociceptive responses by amygdala ERK activation is specific to the persistent inflammatory state. Altogether, our results demonstrate a functional role of the ERK signaling cascade in the amygdala in inflammation-induced peripheral hypersensitivity.

Key words: ERK; inflammatory pain; central nucleus; learning; memory; MAPK; sensitization

\section{Introduction}

The central nucleus of the amygdala (CeA) is well positioned to link noxious stimuli to defense responses. It receives nociceptive information through the spino-parabrachio-amygdaloid pain pathway and polymodal information through the basolateral amygdala (Bernard and Besson, 1990; Bernard et al., 1996; Aggleton, 2000; Neugebauer et al., 2004). The CeA, in turn, projects to the hypothalamus, substantia inominata dorsalis, and various brainstem nuclei that mediate defense behavioral responses (Aggleton, 2000). Numerous anatomical, behavioral, and physiological studies support the role of the amygdala in the modulation of pain perception. Pain-related neural activity changes have been detected in the amygdala in rodents and in humans (Mao et al., 1993; Schneider et al., 2001; Naliboff et al., 2003; Mayer et al., 2005; Berman et al., 2006). In addition, behavioral studies in rodents have demonstrated that the amygdala is necessary for some types of analgesia without affecting responses to acute noxious stimuli (Helmstetter and Bellgowan, 1993; Fox and Sorenson, 1994; Helmstetter et al., 1995; Manning and Mayer, 1995a,b;

\footnotetext{
Received Aug. 15, 2006; revised Jan. 6, 2007; accepted Jan. 9, 2007.

This work was supported by National Institutes of Health Grant NS48602 to Y.C. and R.W.G. We thank D. Sweatt for support and helpful comments on this manuscript.

Correspondence should be addressed to Dr. Robert W. Gereau IV, Washington University Pain Center, Department of Anesthesiology, Washington University School of Medicine, 660 South Euclid Avenue, Campus Box 8054, St Louis, M0 63110. E-mail: gereaur@wustl.edu.

DOI:10.1523/JNEUROSCI.3536-06.2007

Copyright $\odot 2007$ Society for Neuroscience $\quad$ 0270-6474/07/271543-09\$15.00/0
}

Manning, 1998; Crown et al., 2000; Manning et al., 2001, 2003; Shane et al., 2001). Thus, although the amygdala is necessary for antinociception in these models, it does not seem to regulate baseline nociception.

Additional evidence comes from in vivo electrophysiological recordings that have mapped amygdala neurons that respond to peripheral noxious stimuli (Bernard and Besson, 1990; Bernard et al., 1992; Neugebauer and Li, 2002, 2003). Most of these noxious-responsive neurons are located in the laterocapsular division of the central nucleus of the amygdala (CeLC), which is also the target of the spino-parabrachio-amygdaloid pain pathway and a major amygdala output nucleus. Responses of CeLC neurons to noxious stimuli are depressed by systemic morphine administration, further supporting their role in antinociception (Huang et al., 1993). Furthermore, these noxious-responsive neurons in the amygdala undergo persistent changes in excitability and synaptic strength during chronic inflammatory pain conditions, suggesting that endogenous changes in the amygdala might contribute to persistent pain (Neugebauer et al., 2003). Consistent with this hypothesis, intra-amygdala infusion of antagonists of metabotropic glutamate receptor 5 or the CGRP1 receptor decrease pain behaviors in a rat model of arthritis (Han et al., 2005; Han and Neugebauer, 2005).

The extracellular signal-regulated kinases (ERKs) have been shown to modulate synaptic plasticity and amygdala-dependent behavior (Schafe et al., 2000; Lu et al., 2001; Thiels and Klann, 2001; Duvarci et al., 2005; Lu et al., 2005). Because chronic pain 
has been shown to correlate with persistent plasticity in the amygdala, we hypothesized that ERK signaling in the amygdala contributes to long-lasting peripheral hypersensitivity induced by persistent inflammation. We show that ERK is activated in the central nucleus of the amygdala during persistent inflammation, and that blockade of amygdala ERK activation reduces inflammation-induced peripheral tactile hypersensitivity. Furthermore, pharmacological activation of ERK in the amygdala induces bilateral tactile hypersensitivity in the absence of inflammation. Altogether, our results demonstrate that ERK activation in the amygdala is not only necessary, but also sufficient, to induce long-lasting tactile hypersensitivity.

\section{Materials and Methods}

Subjects. Experiments were performed in accordance with the guidelines of the National Institutes of Health and were approved by the Animal Care and Use Committee of Baylor College of Medicine and Washington University School of Medicine. Male Swiss-Webster mice (40-45 g) were housed in a $12 \mathrm{~h}$ light/dark cycle with food and water provided $a d$ libitum.

Surgical procedure. Mice were deeply anesthetized with a combination anesthetic $(2.5 \mathrm{ml} / \mathrm{kg}$ body weight $)$ consisting of ketamine $(37.5 \mathrm{mg} / \mathrm{ml})$, xylazine $(1.9 \mathrm{mg} / \mathrm{ml})$, and acepromazine $(0.37 \mathrm{mg} / \mathrm{ml})$, and were chronically implanted with an $8 \mathrm{~mm}$ 26-gauge stainless-steel guide cannula aimed at the central nucleus of the amygdala (stereotaxic coordinates, 1.4 $\mathrm{mm}$ posterior from bregma, $3.3 \mathrm{~mm}$ lateral to midline, $4.2 \mathrm{~mm}$ ventral from skull surface). The guide cannula was fixed to the skull using two jeweler's screws and dental acrylic. An $8 \mathrm{~mm}$ stylet was placed in the cannula to prevent clogging. Animals recovered for $4 \mathrm{~d}$ before additional experimental procedures. At the end of the experiment, brains were sectioned and Nissl stained to verify cannula position and injection site.

Intra-amygdala drug infusions. Microinjections were performed through a 32-gauge stainless-steel injection cannula that extended 0.5 $\mathrm{mm}$ beyond the tip of the guide cannula. The injection cannula was attached to flexible plastic tubing and a microliter Hamilton syringe to perform the injections. A total volume of $0.3 \mu \mathrm{l}$ was infused over a period of $3 \mathrm{~min}$ and the injection cannula was kept in place for an additional 1 min to allow for drug diffusion. 1,4-Diamino-2,3-dicyano-1,4-bis(2aminophenylthio)butadiene (U0126) was selected to inhibit ERK activation for two main reasons: first, of all of the commercially available mitogenactivated protein kinase kinase (MEK) inhibitors, U0126 offers the best separation between concentrations for specific MEK inhibition versus off-target effects (Favata et al., 1998; Roberson et al., 1999); second, U0126 has the added advantage that it has a commercially available negative structural control analog, 1,4-diamino-2,3-dicyano-1,4-bis(methylthio)butadiene (U0124), which can be used to increase confidence in the MEK/ERKspecificity of the action of the drug. U0126 and U0124 (VWR Scientific Products, Batavia, IL) were dissolved as described previously (Schafe et al., 2000). Drugs were first dissolved in $100 \%$ DMSO to a final stock concentration of $10 \mathrm{~mm}$. On the day of the experiment, U0126 and U0124 were diluted $1: 1$ in $0.9 \%$ saline to a concentration of $5 \mathrm{~mm}$ in $50 \% \mathrm{DMSO} /$ saline. The phorbol ester phorbol 12,13-diacetate was selected to activate ERK because direct activators of ERK are not commercially available. PDA activates PKC (protein kinase $\mathrm{C}$ ), and results in downstream activation of ERK (Roberson et al., 1999; Yuan et al., 2002). PDA (Sigma, St. Louis, MO) was dissolved in $100 \%$ DMSO to a stock concentration of $3 \mathrm{~mm}$. PDA was diluted $1: 1$ in $0.9 \%$ saline to a concentration of $1.5 \mathrm{~mm}$, then diluted to $0.4 \mathrm{~mm}$ with $50 \% \mathrm{DMSO} /$ saline. Fifty percent DMSO/saline was used as a vehicle control for all experiments.

Nociceptive testing. The formalin test was performed as described previously (Karim et al., 2001). Ten microliters of 5\% formalin solution were injected subcutaneously into the plantar surface of the right hindpaw. The time spent in nociceptive behavior, defined as licking, lifting, and flinching of the injected paw, was recorded in $5 \mathrm{~min}$ intervals for $2 \mathrm{~h}$ after the formalin injection, starting immediately after the paw injection. Mechanical sensitivity was measured using von Frey filaments (North Coast Medical, San Jose, CA) as described previously (Yang and Gereau, 2003; Hu et al., 2006). Each von Frey filament, starting with the smallest, was applied to the mouse hindpaw until bent at $\sim 30^{\circ}$ for $\sim 3 \mathrm{~s}$. The smallest filament that evoked a paw withdrawal response in at least three of the five trials was taken as the mechanical threshold. Similar sites were selected for measuring mechanical thresholds in all tested animals and for each individual animal the thresholds were measured at approximately the same site throughout the experiment. Three to five baseline measurements were taken from each hindpaw before formalin injection and the average was calculated individually for each paw as the baseline. Withdrawal thresholds were measured again $3 \mathrm{~h}$ after formalin injection. Mechanical hypersensitivity was calculated as the percentage of baseline for each paw. Thermal sensitivity was measured using a modified version of the Hargreaves test (Hargreaves et al., 1988) as described previously (Bhave et al., 2001). Animals were placed in a Plexiglas testing chamber on an elevated platform with a clear glass surface heated to $30^{\circ} \mathrm{C}$. A thermal stimulus from a constant radiant heat source with an active intensity of $25 \%$ (active intensity is the intensity of light source as defined by the manufacturer) was delivered through the glass bottom of the chamber to the plantar surface of the hind paw (IITC Life Sciences, Woodland Hills, CA). The latency for foot withdrawal was measured. Three to five baseline latencies were taken from each hindpaw before formalin injection and the average was calculated individually for each paw as the baseline. Withdrawal latencies were measured again $3 \mathrm{~h}$ after formalin injection. Thermal hypersensitivity was calculated as the percentage of baseline for each paw. For the U0126 experiments, intraamygdala drug infusions were performed $2 \mathrm{~h}$ after formalin injection and mechanical or thermal thresholds were measured $1 \mathrm{~h}$ after intraamygdala drug infusion, which corresponds to $3 \mathrm{~h}$ after formalin injection in the paw. The effects of U0126 on baseline mechanical thresholds were measured $1 \mathrm{~h}$ after intra-amygdala drug infusion. To measure the effects of U0126 on formalin-induced spontaneous nociceptive behavior, intra-amygdala drug infusions were performed $30 \mathrm{~min}$ before formalin injection. The effects of PDA on baseline mechanical thresholds were measured $30 \mathrm{~min}$ after drug infusion. All behavioral testing was performed blind to pharmacological treatment.

Sample preparation. At 3, 25, and $180 \mathrm{~min}$ after formalin injection, brains were removed and sectioned into 2 -mm-thick coronal sections using an acrylic brain matrix (Stoelting, Wood Dale, IL). From the appropriate section, amygdala punches were obtained using a custommade $1 \mathrm{~mm}$ punch tool. Samples were homogenized in ice-cold homogenization buffer (20 mm Tris-HCl, pH 7.5, 1 mm EDTA, 1 mm sodium pyrophosphate, $25 \mu \mathrm{g} / \mathrm{ml}$ aprotinin, $25 \mu \mathrm{g} / \mathrm{ml}$ leupeptin, $1 \mu \mathrm{g} / \mathrm{ml} \mathrm{micro-}$ cystein, $1 \mathrm{~mm}$ sodium orthovanadate, and $100 \mu \mathrm{m}$ phenylmethylsulphonyl fluoride; Sigma) using a Dounce homogenizer. Protein concentrations were determined using the BCA Protein Assay kit (Pierce Biotechnology, Rockford, IL). For the U0126 experiments, samples were collected $1 \mathrm{~h}$ after intra-amygdala drug infusion, which corresponds to $3 \mathrm{~h}$ after formalin paw injection.

Western blotting. The levels of formalin-induced ERK activation in the amygdala were measured by Western blotting using antibodies specific for phosphorylated (active) ERK or for total ERK. Total protein $(10 \mu \mathrm{g})$ from amygdala homogenates was electrophoresed in 10\% SDS polyacrylamide gels and blotted to nitrocellulose membranes. All incubations were at room temperature. Blots were blocked in 3\% bovine serum albumin (BSA) in Tris-buffered saline and Tween 20 (TBST; 50 mm Tris-HCl, $\mathrm{pH} 7.5 ; 150 \mathrm{~mm} \mathrm{NaCl} ; 0.05 \%$ Tween 20 ) for $1 \mathrm{~h}$ and then incubated in antiphospho-p44/42 ERK or anti-p44/42 ERK primary antibody (1:1000 in 3\% BSA/TBST; Cell Signaling Technology, Beverly, MA) for $1 \mathrm{~h}$. After several washes, blots were incubated in biotinylated secondary antibody (1:5000 in 3\% BSA/TBST; Millipore, Temecula, CA) for $1 \mathrm{~h}$, then washed and incubated in $\mathrm{I}^{125}$-Streptavidin (1:500 in TBST; Amersham Biosciences, Arlington Heights, IL) for $1 \mathrm{~h}$. Blots were washed and exposed to a PhosphorImager screen. Densitometry of bands corresponding to ERK1 (p44) and ERK2 (p42) was performed using Optiquant software (Packard Instruments, Meridian, CT).

Immunohistochemistry. Three hours after formalin injection into the paw, mice were deeply anesthetized with $1.25 \%$ Avertin anesthesia (2,2,2-tribromoethanol and tert-amyl alcohol in $0.9 \% \mathrm{NaCl} ; 0.025 \mathrm{ml} / \mathrm{g}$ body weight), then perfused transcardially with $0.9 \% \mathrm{NaCl}\left(37^{\circ} \mathrm{C}\right)$, followed by $300 \mathrm{ml}$ of ice-cold $4 \%$ paraformaldehyde solution in $0.1 \mathrm{M}$ 
phosphate buffer (PB), pH 7.4. The brain was dissected and postfixed in $4 \%$ paraformaldehyde $/ \mathrm{PB}$ for $4 \mathrm{~h}$ at $4^{\circ} \mathrm{C}$, followed by cryoprotection in $30 \%$ sucrose $/ \mathrm{PB}$ for $48 \mathrm{~h}$ at $4^{\circ} \mathrm{C}$. Coronal sections $(30 \mu \mathrm{m})$, obtained using a freezing sliding microtome, were collected in ice-cold PB. All other incubations were at room temperature. Sections were rinsed in $10 \%$ methanol $0.3 \% \mathrm{H}_{2} \mathrm{O}_{2}$ in $0.1 \mathrm{M}$ PBS, pH 7.4, for $30 \mathrm{~min}$, then blocked in $1 \%$ normal goat serum with $0.2 \%$ Triton X-100 (NGST) for $1 \mathrm{~h}$. Sections were incubated overnight at $4^{\circ} \mathrm{C}$ in anti-phospho-p44/42 ERK antibody (1:500 in 1\% NGST; Cell Signaling Technology). Sections were then rinsed in $1 \%$ NGST, then incubated in a biotinylated secondary antibody (1:200 in 1\% NGST; Vector Laboratories, Burlingame, CA) for $90 \mathrm{~min}$. After rinsing, the sections were incubated in ExtraAvidin peroxidase (1:1000 in 1\% NGST; Sigma) for $1 \mathrm{~h}$. Detection was performed using a DAB Substrate kit (Vector Laboratories). Sections were mounted on glass slides, air-dried, dehydrated, and coverslipped with distrene plasticizer xylene mounting medium. For the U0126 experiments, samples were collected $1 \mathrm{~h}$ after intra-amygdala drug infusions, and for the PDA experiments, 30 min after intra-amygdala drug infusions. PhosphoERK immunostainings were only used to qualitatively examine the anatomical distribution of ERK activation. Comparison between conditions was performed by simple observation of immunostaining experiments performed on the same day and under the same experimental conditions.

\section{Results}

ERK is activated in the central nucleus of the amygdala during persistent peripheral inflammation

To test whether ERK activation in the amygdala modulates longlasting peripheral hypersensitivity during persistent inflammation, we used the formalin test as a model of inflammatory pain in mice (Tjolsen et al., 1992). Subcutaneous injection of dilute formalin (5\%, $10 \mu \mathrm{l}$ ) into the plantar surface of the hindpaw induced inflammation and an immediate spontaneous behavioral response that included licking, lifting, and flinching of the injected paw. Formalin injection produced a triphasic behavioral response with an early peak at $5 \mathrm{~min}$ after the injection, an intermediate peak at $25 \mathrm{~min}$, and a late phase occurring between $60 \mathrm{~min}$ and $2 \mathrm{~h}$ after the formalin injection (Fig. 1a). Saline injection did not produce significant nociceptive responses during the $2 \mathrm{~h}$ of observation (Fig. $1 a$ ).

In addition to the spontaneous nociceptive behavioral responses, formalin injection into the paw produced long-lasting hypersensitivity in both the injected paw and the noninjected hindpaw. Formalin injection decreased thermal withdrawal latencies from $10.06 \pm 0.71 \mathrm{~s}$ to $5.21 \pm 0.57 \mathrm{~s}$ (mean $\pm \mathrm{SE} ; n=6$ animals; $p<0.0001$; $~ 50.98 \%$ of baseline) in the injected paw, and from $11.83 \pm 0.81 \mathrm{~s}$ to $7.83 \pm 1.25 \mathrm{~s}$ (mean $\pm \mathrm{SE} ; n=6$ animals; $p=0.0058 ; \sim 65.35 \%$ of baseline) in the noninjected hindpaw (Fig. 1b). Similarly, injecting formalin in the paw decreased mechanical thresholds from $2.07 \pm 0.45 \mathrm{~g}$ to $0.24 \pm$ $0.08 \mathrm{~g}$ (mean $\pm \mathrm{SE} ; n=6$ animals; $p=0.0064 ; \sim 12 \%$ of baseline) in the injected paw, and from $2.10 \pm 0.40 \mathrm{~g}$ to $1.00 \pm 0.15 \mathrm{~g}$ (mean \pm SE; $n=6$ animals; $p=0.0205 ; \sim 51 \%$ of baseline) in the noninjected hindpaw (Fig. 1c). No significant changes in either mechanical or thermal thresholds were observed after saline injection (Fig. 1b,c).

To test whether persistent peripheral inflammation induces ERK activation in the amygdala, we examined the levels of ERK activation in the amygdala at different time-points after formalin or saline injection in the paw by Western blotting using antibodies specific for phosphorylated (active) ERK or total ERK. Increased ERK activation was observed in the amygdala $3 \mathrm{~h}$ after formalin injection, when mice exhibit mechanical and thermal hypersensitivity, but not at earlier time points (3 and $25 \mathrm{~min}$ ) when animals engage in spontaneous nociceptive behaviors (Fig. $2 a, b)$. A significant increase in ERK activation was seen in the right amygdala for the p42 (ERK2) but not the p44 (ERK1) iso-
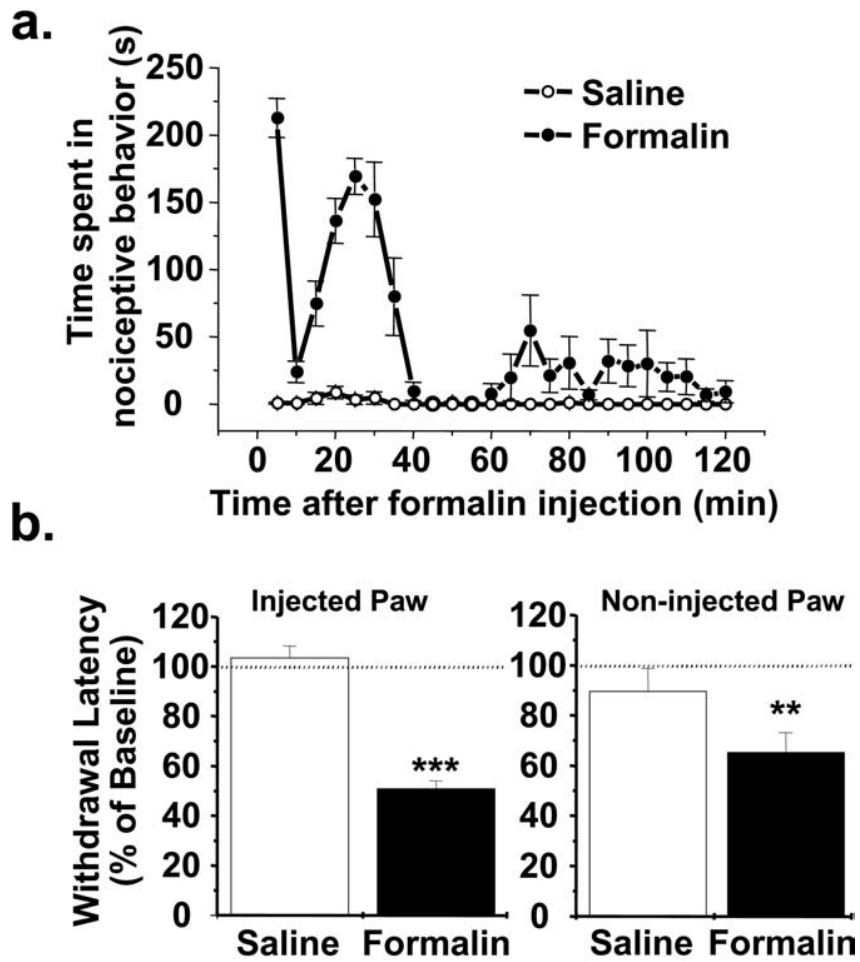

C.

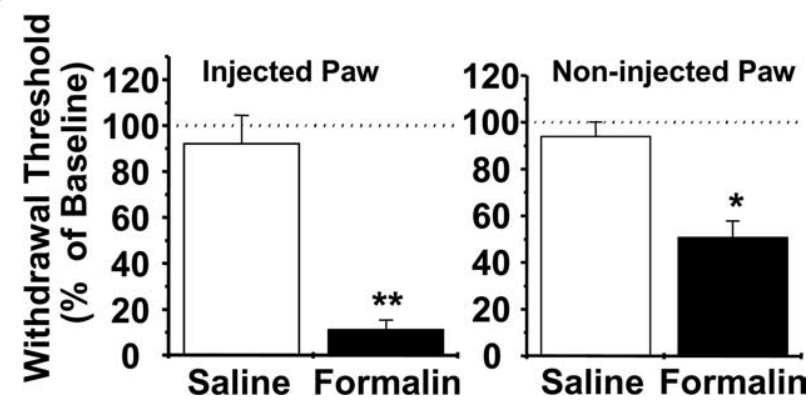

Figure 1. Formalin-induced nociceptive behavior. $\boldsymbol{a}$, Time course of formalin-induced spontaneous nociceptive behavior. Formalin injection induced triphasic spontaneous nociceptive behavior lasting for $2 \mathrm{~h}$ after injection ( $n=9$ animals for formalin treatment and 3 animals for saline treatment). $\boldsymbol{b}$, Three hours after formalin injection, thermal latencies in the injected and noninjected hindpaw decreased to $51 \%\left({ }^{* * *} p<0.0001\right)$ and to $65 \%$ of baseline $\left({ }^{* *} p=\right.$ 0.0058 ), respectively, in formalin-injected but not saline-injected mice ( $n=6$ animals per treatment). c, Three hours after formalin injection, mechanical thresholds of the injected and noninjected paw decreased to $12 \%\left({ }^{* *} p=0.0064\right.$, paired $t$ test) and to $51 \%$ of baseline, respectively, ${ }^{*} p=0.0205$, paired $t$ test $)$ in formalin-injected but not saline-injected mice $(n=$ 6 animals per treatment). Error bars indicate SEM.

form (Fig. 2b). Interestingly, ERK activation was seen in the right amygdala whether we injected formalin in the right or the left hindpaw. Right paw injection was used for the remainder of the experiments. No significant changes in the levels of total ERK were observed for either the p42 (ERK2) or the p44 (ERK1) isoform. Immunostaining using antibodies specific for the active form of ERK revealed that formalin-induced ERK activation is almost exclusively localized to the laterocapsular division of the CeLC, where intense pERK staining was observed in neuronal somata and processes (Fig. 2c).

Blockade of ERK activation in the amygdala reduces inflammation-induced peripheral hypersensitivity

The strong ERK activation in the CeLC $3 \mathrm{~h}$ after formalin injection suggested that this activation might underlie inflammation- 
induced long-lasting hypersensitivity, but not spontaneous nociceptive responses to formalin or baseline nociception. To test this hypothesis, we inhibited ERK activation in the amygdala by locally infusing the MEK inhibitor, U0126, and then measured the effects of the drug on formalininduced ERK activation and nociception. Typical infusion sites are shown in Figure $3 b$. To determine the concentration of U0126 that inhibits formalin-induced ERK activation in the amygdala, we infused U0126 into the amygdala in doses ranging from 0.15 to $1.5 \mathrm{nmol}$. Western blots for phospho-ERK showed that the MEK inhibitor U0126, but not its structural analog control U0124, inhibited formalin-induced amygdala ERK activation in a dose-dependent manner (Fig. $3 a$ ). We selected the $1.5 \mathrm{nmol}$ dose for the behavioral experiments because it was the lowest dose that significantly inhibited formalin-induced ERK activation.

Immunostaining on coronal brain sections revealed a clear inhibition of ERK activation in the CeA by U0126 when compared with control U0124-infused mice (Fig. 3b). Offsite infusion of U0126 did not inhibit amygdala ERK activation (Fig. $3 b$ ). In addition, immunostaining on sagittal sections from brains of U0126infused mice showed a localized action of U0126, with inhibition of ERK activation restricted to the injection site only; phospho-ERK immunopositive cells can be clearly identified anterior and posterior to the injection site (Fig. 3c). To further confirm the extent of ERK inhibition by U0126, we injected the phorbol ester PDA at concentrations sufficient to activate ERK in the entire amygdala (450 pmol), and then paired this with the infusion of U0126 (1.5 nmol in $0.3 \mu \mathrm{l}$ ). Immunostaining for phospho-ERK in brain slices taken from these animals revealed that inhibition of ERK activation by U0126 is restricted to the CeA, with obvious significant ERK activation still present in other adjacent amygdala nuclei including the lateral nucleus and basolateral nucleus (Fig. 3d).

To test whether blocking ERK activation in the amygdala has an effect on spontaneous formalin-induced behaviors, we infused vehicle (50\% DMSO) or U0126 (1.5 nmol) into the amygdala $30 \mathrm{~min}$ before injection of formalin into the hindpaw. Blinded behavioral analysis revealed that spontaneous nociceptive responses to formalin were not affected by inhibition of ERK activation by U0126 (Fig. 4a).

To test whether the amygdala ERK activation observed $3 \mathrm{~h}$ after formalin injection mediates thermal and tactile hypersensitivity observed at the same time-point, we compared the effects of intra-amygdala infusion of U0126 (1.5 nmol), U0124 (1.5 nmol), or vehicle (50\% DMSO) on thermal and mechanical hypersensi- b.
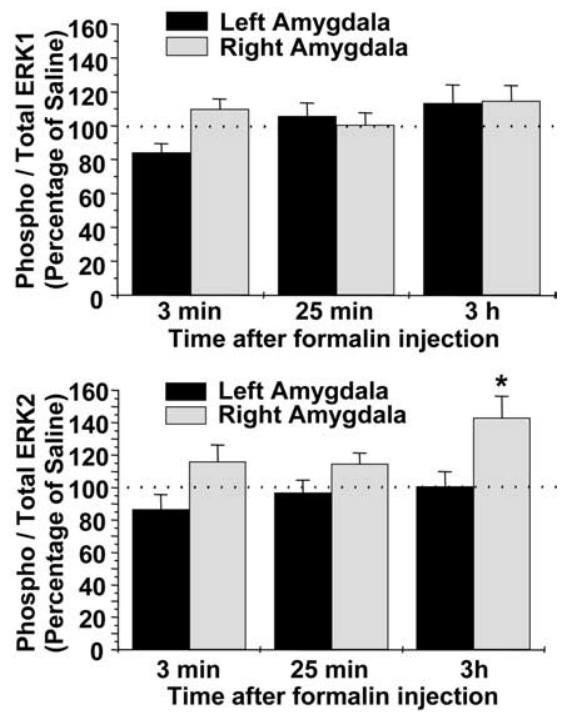

3 hours

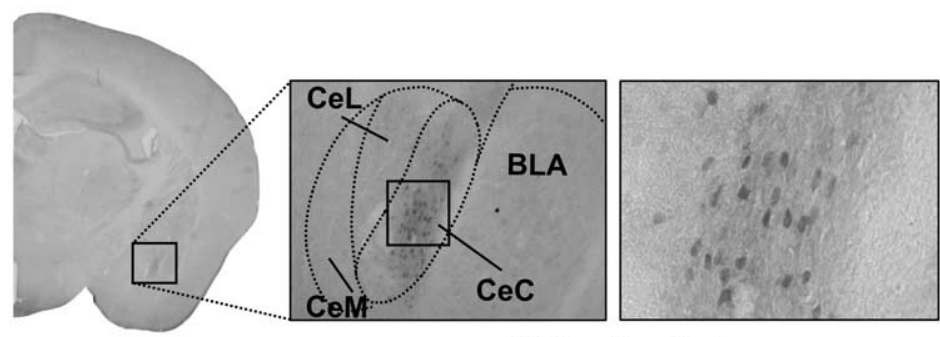

Saline Treated

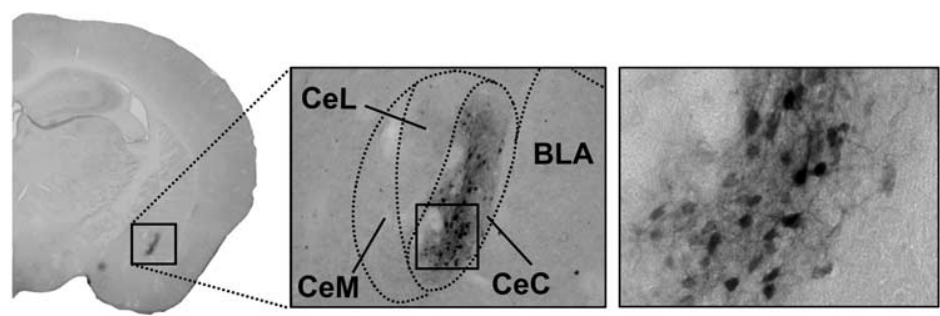

Formalin Treated

Figure 2. Formalin-induced amygdala ERK activation. $\boldsymbol{a}$, Representative Western blots for phospho-ERK (top) and total-ERK (bottom) $3 \mathrm{~h}$ after formalin or saline paw injection. $\boldsymbol{b}$, Densitometry analysis of bands corresponding to ERK1 (p44) and ERK2 (p42) show that ERK2 activation in the amygdala of formalin-injected animals is significantly greater than in saline-injected animals $3 \mathrm{~h}$, but not 3 min or 25 min after formalin injection ( $n=10$ animals per treatment per time point; ${ }^{*} p<0.05$, two-way ANOVA, Bonferroni's post hoc test). No significant ERK1 activation was observed. c, Anatomical distribution of formalin-induced ERK (top) or formalin (bottom) injection in the hindpaw. Formalin-induced ERK activation is localized to the laterocapsular subdivision 列 subdivision of central nucleus; $\mathrm{CeC}$, capsular subdivision of central nucleus; CeM, medial subdivision of central nucleus; BLA, basolateral nucleus. Error bars indicate SEM.

tivity observed $3 \mathrm{~h}$ after formalin injection. Blinded behavioral analysis revealed that inhibition of amygdala ERK activation by U0126 did not affect thermal hypersensitivity in either the injected or the noninjected hindpaw, but significantly decreased mechanical hypersensitivity both in the injected and in the noninjected hindpaw compared with animals infused in the amygdala with vehicle or the structural analog U0124 (Fig. 4c,d). Thus, mechanical thresholds in the injected paw of U0126-infused mice were $0.73 \pm 0.14 \mathrm{~g}$ (mean $\pm \mathrm{SE} ; \sim 57 \%$ of baseline), whereas vehicle- and U0124-infused mice had mechanical thresholds of $0.11 \pm 0.03 \mathrm{~g}$ and $0.18 \pm 0.05 \mathrm{~g}$ (mean $\pm \mathrm{SE} ; \sim 11 \%$ of baseline) respectively. Similar effects on mechanical thresholds were ob- 


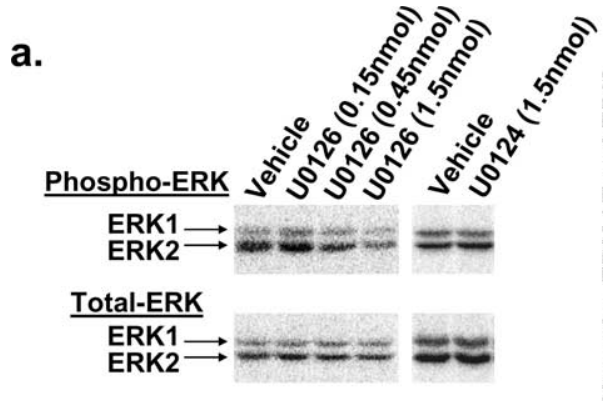

b.

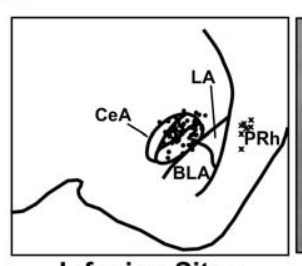

Infusion Sites

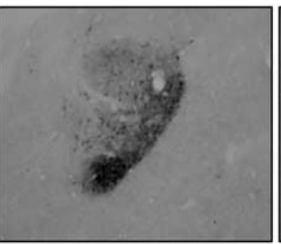

Control (U0124)

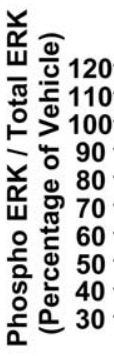

Vehicle 0.15
U0126 (nmoles)

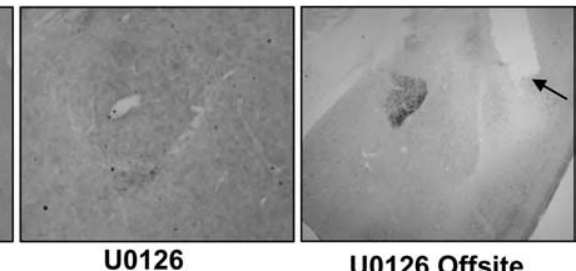

U0126 Offsite c.

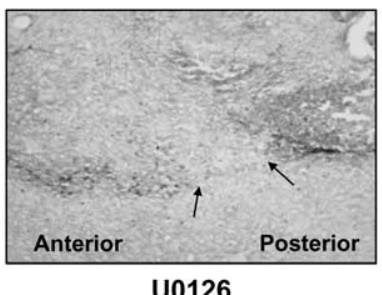

d.

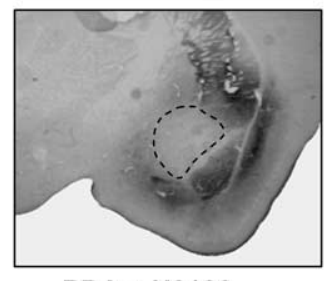

PDA + U0126

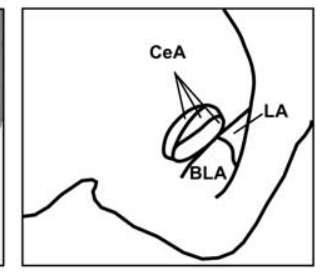

Figure 3. Inhibition of formalin-induced amygdala ERK activation by U0126. $a$, Representative Western blots for phospho-ERK and total-ERK $1 \mathrm{~h}$ after intra-amygdala drug infusion (left). U0126 was infused into the amygdala in doses ranging from 0.15 to 1.5 nmol in a total volume of $0.3 \mu$ l. Densitometry analysis of bands corresponding to ERK1 (p44) and ERK2 (p42) is shown in the right panel. U0126 inhibits phosphorylation of ERK in a dose-dependent manner ( $n=3$ animals; ${ }^{*} p<0.05$, one-way ANOVA, Tukey's post hoc test). The asterisk indicates significant inhibition of ERK activation by U0126 compared with vehicle-treated animals. $\boldsymbol{b}$, The first panel (left) is a diagram of a coronal section showing typical injection sites into the central nucleus of the amygdala (solid circles) or offsite injection sites into the perirhinal cortex (x). LA, Lateral nucleus; BLA, basolateral nucleus; PRh, perirhinal cortex. The second, third, and fourth panels are immunohistochemistry for phospho-ERK on coronal brain sections taken from animals after intra-amygdala or offsite drug infusion. U0126 (third panel), but not the control structural analog U0124 (second panel), inhibits formalin-induced amygdala ERK activation. Offsite infusion of U0126 (fourth panel) did not affect formalin-induced amygdala ERK activation. The tip of the injector is indicated by the arrow. c, Immunohistochemistry for phospho-ERK on a sagittal section. The arrows indicate the extent of ERK inhibition by U0126, with phospho-ERK immunopositive cells anterior and posterior to the injection site. $\boldsymbol{d}$, Coinfusion of PDA with U0126; a diagram depicting the anatomical localization of different amygdala nuclei is shown in the right panel. Inhibition of PDA-induced ERK activation by U0126 is restricted to the CeA; U0126 extent of inhibition is delineated by the dotted line (left). Error bars indicate SEM.

served in the noninjected hindpaw, with a mean threshold of $1.07 \pm 0.12 \mathrm{~g}$ (mean $\pm \mathrm{SE} ; \sim 85 \%$ of baseline) in U0126-infused mice and $0.59 \pm 0.17 \mathrm{~g}$ and $0.63 \pm 0.12 \mathrm{~g}$ (mean $\pm \mathrm{SE} ; \sim 49 \%$ of baseline) in vehicle- and U0124-infused mice respectively. Mechanical and thermal thresholds in the injected and noninjected hindpaw of U0124-infused mice were not significantly different from thresholds in vehicle-infused mice. The effects of U0126 were specific to the amygdala as offsite infusion of U0126 did not affect formalin-induced mechanical hypersensitivity (Fig. 4e). Moreover, these effects of U0126 on mechanical hypersensitivity were not attributable to effects on baseline mechanical sensitivity because infusion of U0126 did not significantly affect baseline mechanical thresholds when compared with vehicle-infused mice (Fig. 4b).

Altogether, our behavioral results show that inhibition of ERK

activation in the amygdala does not affect baseline nociception in the absence of inflammation, spontaneous nociceptive responses to formalin, or formalin-induced thermal hypersensitivity, but significantly reduces formalin-induced mechanical hypersensitivity. These results nicely correlate with our biochemical data that show significant ERK activation in the amygdala $3 \mathrm{~h}$ after formalin injection, but not at earlier time points (Fig. $2 b$ ). The lack of modulation of inflammation-induced thermal hypersensitivity (which is also observed $3 \mathrm{~h}$ after inflammation when ERK is activated in the amygdala) by inhibition of amygdala ERK activation suggests that modulation of hypersensitivity by ERK in the amygdala depends on the modality of the nociceptive input.

\section{Pharmacological activation of ERK in the amygdala induces bilateral peripheral hypersensitivity in the absence of inflammation}

So far, we have demonstrated that ERK is activated in the central nucleus of the amygdala during peripheral inflammation and that this ERK activation is necessary for inflammation-induced bilateral mechanical hypersensitivity. To further examine the role of amygdala ERK activation in nociception, we asked whether ERK activation in the amygdala would be sufficient to induce peripheral hypersensitivity in the absence of inflammation.

We mimicked the inflammationinduced ERK activation in the amygdala by infusing the phorbol ester PDA. To determine the concentration of PDA that locally activates ERK in the amygdala, we infused PDA into the amygdala in doses ranging from 45 to 450 pmol. Immunostaining for phospho-ERK revealed that infusion of PDA into the amygdala resulted in a robust dose-dependent activation of ERK, with the extent of ERK activation increasing with increasing doses of PDA (Fig. 5a). We chose the 120 pmol dose for our behavioral experiments because it was the lowest dose of PDA that locally activated ERK in the amygdala.

To test whether pharmacological activation of ERK in the amygdala is sufficient to induce peripheral hypersensitivity in the absence of inflammation, we measured the effects of infusing PDA (120 pmol) into the amygdala on baseline mechanical and thermal thresholds. Blinded behavioral analysis revealed that infusion of PDA into the amygdala did not affect baseline thermal sensitivity but significantly decreased mechanical thresholds of both the right and left hindpaw (Fig. 5b,c). PDA-induced peripheral tactile hypersensitivity was ERK-dependent because it was prevented by coinfusion of the MEK inhibitor, U0126 (1.5 nmol) (Fig. 5c). Our results demonstrate that pharmacological activation of ERK in the amygdala induces bilateral hypersensitivity to tactile stimulation that mimics inflammation-induced hypersen- 
a.

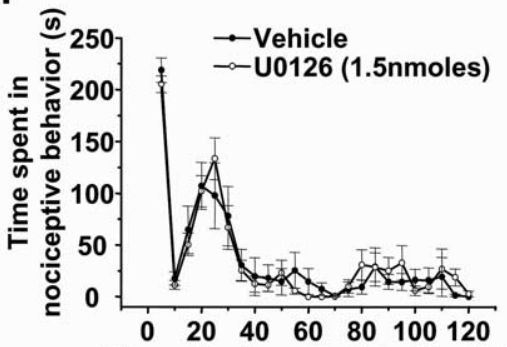

c.

d.
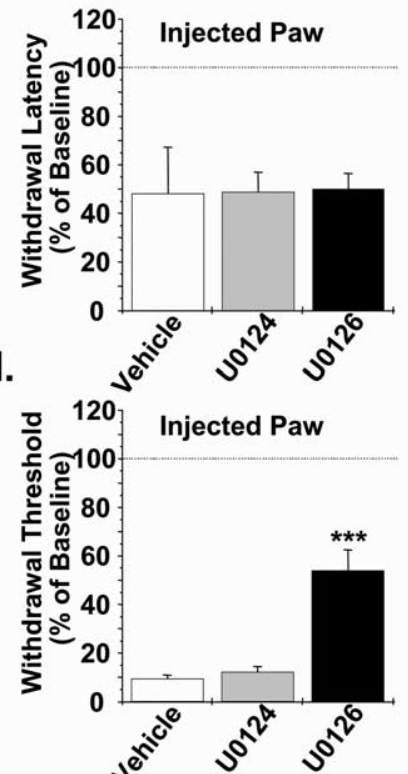

e.

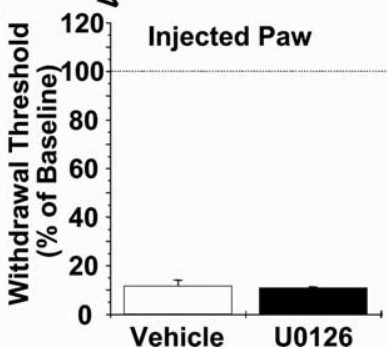

b.
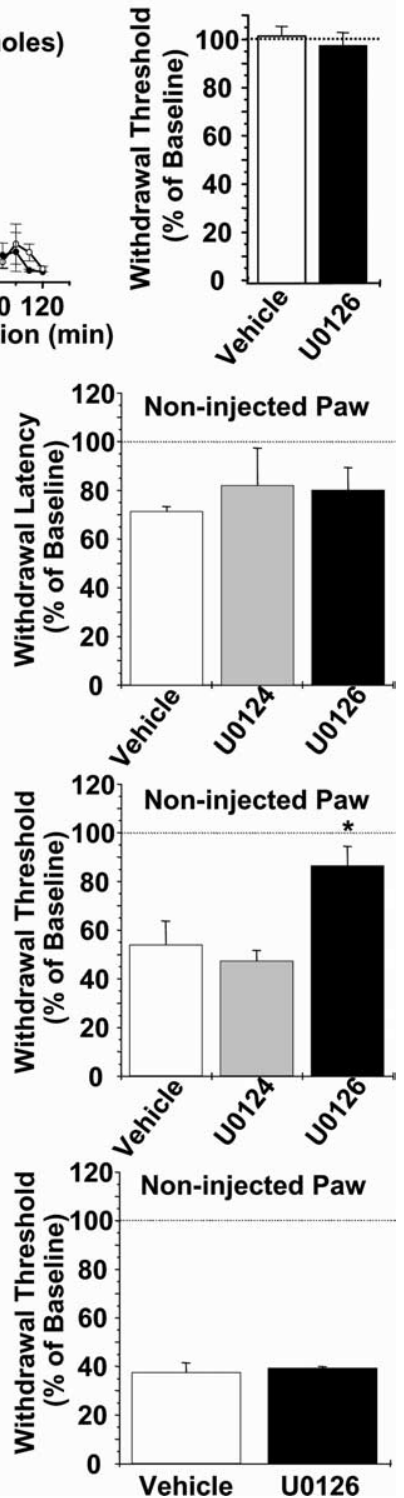

Figure 4. Effects of intra-amygdala infusion of U0126 on nociceptive behavior. $\boldsymbol{a}$, Spontaneous nociceptive responses to formalin were not significantly different in U0126-versus vehicle-injected mice ( $n=8-10$ animals per treatment). $\boldsymbol{b}$, Baseline mechanical thresholds (in the absence of inflammation) are not affected by intra-amygdala infusion of vehicle $(50 \%$ DMSO) or U0126 (1.5 nmol) ( $n=3$ mice per treatment). $\boldsymbol{c}, \boldsymbol{d}$, Five days after cannulation, animals were injected with $5 \%$ formalin into the hindpaw. Two hours after the paw injection, the MEK inhibitor U0126, the structural analog control compound U0124, or vehicle was infused into the amygdala. One hour after the amygdala injection, the effects of these treatments on thermal latencies $(\boldsymbol{c})$ or mechanical thresholds (d) were analyzed. $\boldsymbol{c}$, U0126 infusion into the amygdala did not affect formalin-induced thermal hypersensitivity $(n=4$ animals per treatment). $\boldsymbol{d}$, U0126 infusion significantly reduced formalin-induced mechanical hypersensitivity in both the injected and the noninjected hindpaw ( $n=6-7$ animals per treatment; $p<0.05$, one-way ANOVA, Tukey's post hoc test, $\left.{ }^{*} p<0.05 ;{ }^{* * *} p<0.0001\right)$. e, Offsite infusion of U0126 $(1.5 \mathrm{nmol})$ did not affect formalin-induced mechanical hypersensitivity $(n=3$ animals per treatment). Error bars indicate SEM.

sitivity, but occurs in the absence of inflammation. The lack of modulation of thermal sensitivity by PDA is consistent with our inhibitor data and supports the hypothesis that modulation of hypersensitivity by ERK in the amygdala depends on the modality of the nociceptive input.

a. Phospho-ERK Immunostaining after PDA Infusion

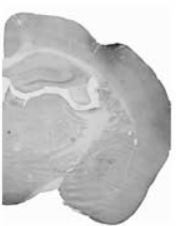

45 pmol

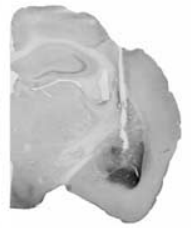

$120 \mathrm{pmol}$

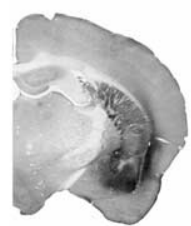

225 pmol

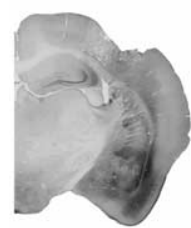

$450 \mathrm{pmol}$ b.
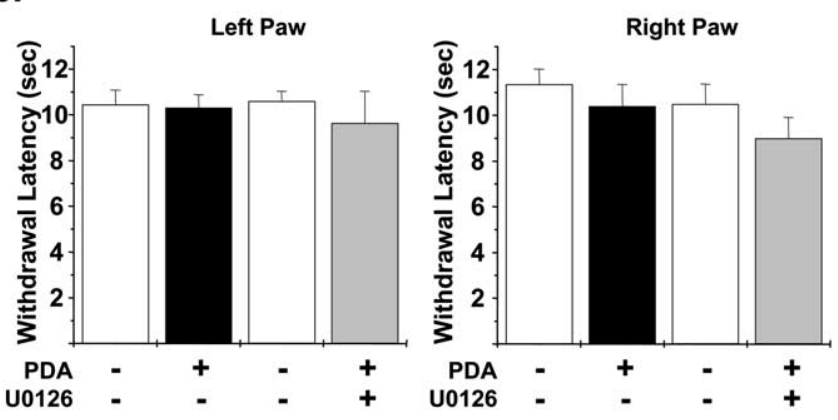

c.
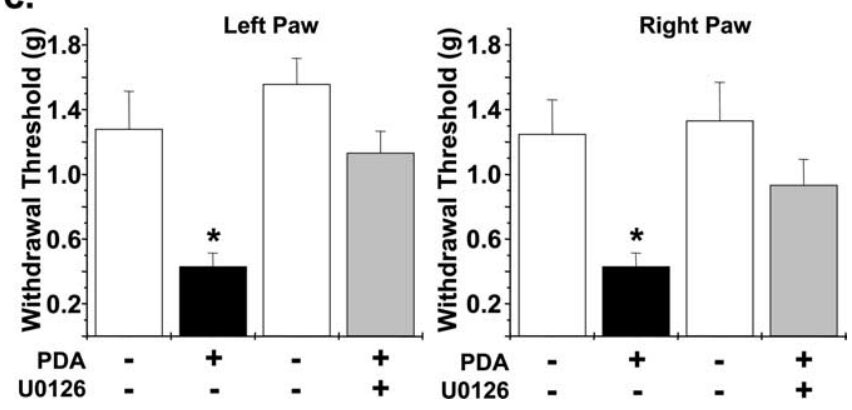

Figure 5. PDA-induced ERK activation in the amygdala and effects on mechanical and thermal thresholds in the absence of inflammation. Five days after cannulation, mice received an infusion of the phorbol ester PDA (doses ranging from 45 to $450 \mathrm{pmol}$ ), or a coinfusion of PDA (120 pmol) plus U0126 (1.5 nmol) into the amygdala. In all cases, the total infusion volume was $0.3 \mu \mathrm{l} . \boldsymbol{a}$, Extent of ERK activation by various doses of PDA. Infusion of PDA resulted in a robust dose-dependent increase in ERK activation in the amygdala. $\boldsymbol{b}$, PDA (120 pmol) infusion into the amygdala did not affect baseline thermal thresholds in either the right or the left hindpaw ( $n=$ 6-7 animals per treatment). c, PDA (120 pmol) infusion into the amygdala decreased mechanical thresholds of both the right and the left hindpaw. PDA-induced behavior was blocked by coinfusion of PDA with U0126 ( $n=5-6$ animals; one-way ANOVA, Tukey's post hoc test, ${ }^{*} p<$ 0.05). Error bars indicate SEM.

\section{Discussion}

The evidence in the present study confirms that endogenous molecular changes in the amygdala mediate a major component of tactile hypersensitivity induced by persistent inflammation. We show that ERK activation in the amygdala is necessary and sufficient for the behavioral expression of peripheral tactile hypersensitivity, because blockade of amygdala ERK activation decreased inflammation-induced tactile hypersensitivity and pharmacological activation of ERK induced tactile hypersensitivity in the absence of tissue injury. These results strongly support the hypothesis that neuromodulation in the amygdala underlies a component of persistent pain. The ability of amygdala ERK activation to induce tactile hypersensitivity in the absence of tissue injury suggests that ERK activation in the amygdala might be a general mechanism underlying persistent tactile hypersensitivity. Forebrain modulation of pain independent of peripheral injury or inflammation could underlie certain chronic pain conditions that can outlast the initial pathology. 
Blocking ERK activation did not affect baseline nociception in the absence of inflammation, suggesting that blockade of ERK activation in the amygdala does not interfere with the important protective role of acute pain. Spontaneous behavioral responses to formalin were also unaffected by inhibition of ERK activation, further supporting the role of amygdala ERK activation in longlasting hypersensitivity but not acute nociception. Our observations are consistent with previous lesion and chemical inactivation studies, which show that the amygdala does not modulate baseline nociception (Helmstetter and Bellgowan, 1993; Fox and Sorenson, 1994; Manning and Mayer, 1995a; Jasmin et al., 2003) or spontaneous responses to formalin (Manning and Mayer, 1995b; Manning, 1998; Gao et al., 2004).

Our previous studies showed that dominant-negative (DN) MEK mutant mice show reduced formalin-induced spontaneous behavior (Karim et al., 2006), whereas our present data that show no effect of MEK inhibitors on formalin-induced spontaneous behavior. Our previous study used DN MEK mutant mice in which MEK function is suppressed in all neurons in the CNS. In the present study, we locally suppressed MEK function only in the amygdala. Our results therefore suggest that the reduced formalin-induced spontaneous behavior observed in the DN MEK mutant mice is not mediated by effects in the amygdala, but it is likely mediated by suppression of MEK function in the spinal cord or other brain regions.

Several lines of evidence suggest that the amygdala may be involved in both ascending and descending nociceptive pathways. An ascending spino-parabrachio-amygdaloid pain pathway has been described previously (Bernard et al., 1989; Bernard and Besson, 1990; Jasmin et al., 1997), originating in lamina I of the spinal cord and medullary dorsal horn and relaying in the parabrachial area to terminate in the CeA. Our experiments show that inflammation-induced amygdala ERK activation is specifically localized to the laterocapsular division of the CeA, which is also the target of the spino-parabrachio-amygdaloid ascending pain pathway (Bernard et al., 1989; Bernard and Besson, 1990). These results suggest that formalin-induced amygdala ERK activation might occur through the activation of the spinoparabrachio-amygdaloid pathway.

The capability of the amygdala to produce and modulate analgesia suggests that the amygdala is not only part of ascending pain pathways but that it is also involved in descending modulation. Infusion of opioid agonists into the amygdala results in analgesia that is expressed through the periaqueductal gray (PAG) and the rostral ventromedial medulla (Helmstetter et al., 1998; McGaraughty and Heinricher, 2002; Shane et al., 2003), two brainstem structures that are part of descending pain pathways with well established roles in pain modulation. In addition, increased neural transmission in the CeA-PAG pathway during long-term stress has been described previously in rats (Adamec et al., 2003), further supporting the existence of a functional pathway between the CeA and the PAG. The decreased tactile hypersensitivity observed after blocking amygdala ERK activation and the ERK-induced tactile hypersensitivity that occurs in the absence of inflammation suggests that descending pain pathways, such as the CeA-PAG pathway, might be modulated by amygdala ERK signaling and might underlie persistent pain. Descending pain pathways can be both inhibitory and facilitatory; whether ERK activation in the amygdala modulates descending inhibition or facilitation cannot be gleaned from the present study. The peripheral hypersensitivity induced by amygdala ERK activation could be explained by either a net activation of facilitatory path- ways, a net inhibition of descending inhibitory pathways, or the modulation of both.

Our data indicate that ERK is activated in the right amygdala, independent of the side of peripheral inflammation. These results were initially surprising given the numerous anatomical studies that have demonstrated that ascending pain pathways, including the spino-parabrachio-amygdaloid pathway, carry information from the contralateral side of the body. In contrast, in vivo electrophysiological recordings that mapped amygdala neurons that respond to peripheral noxious stimuli show that the receptive fields of these noxious-responsive neurons include areas from the ipsilateral and the contralateral side of the body (Bernard and Besson, 1990; Bernard et al., 1992; Neugebauer and Li, 2002, 2003). In addition, hemispheric lateralization of amygdala function has been reported in humans and in rodents (ColemanMesches and McGaugh, 1995; Morris et al., 1998; Phelps et al., 2001; Baker and Kim, 2004; Cahill et al., 2004; Lee et al., 2004). Therefore, our results showing unilateral ERK activation independent of the stimulation side are not without support. It is also important to mention that the CeLC has been shown to receive a bilateral projection from the parabrachial nucleus, suggesting that both the right and left CeLC receive nociceptive inputs from the spino-parabrachio-amygdaloid pathway (Bernard et al., 1993). Whereas this detailed anatomical study by Bernard et al. (1993) clearly demonstrates that both CeLC receive inputs from the parabrachial nucleus, our results suggest that these anatomical connections might not be identical but might be differentially modulated by a still unknown mechanism. Additional investigation is necessary to determine whether the hemispheric lateralization that we observe in the present study is functionally significant, if it is specific to ERK, and if it is specific to this particular pain model.

Our data indicate that inhibition of ERK activation in the amygdala selectively decreases tactile but not thermal hypersensitivity. Similarly, pharmacological activation of ERK in the amygdala resulted in tactile but not thermal hypersensitivity in the absence of peripheral inflammation. These results demonstrate that modulation of hypersensitivity by ERK activation in the amygdala depends on the modality of the nociceptive input. The difference in modulation of mechanical and thermal hypersensitivity by amygdala ERK activation could thus result from activation of different ascending pathways for the two stimulus modalities. At the same time, the lack of effect of pharmacological ERK activation on thermal sensitivity suggests that this difference does not rely solely on the input side because exogenous activation of ERK in the amygdala did not affect thermal sensitivity. A simple explanation for the lack of modulation of thermal hypersensitivity by amygdala ERK activation is that perhaps the amygdala does not modulate thermal hypersensitivity. CeA neurons respond to acute peripheral thermal and mechanical stimulation (Bernard et al., 1992; Neugebauer and Li, 2002); however, after prolonged pain, amygdala responsiveness to peripheral thermal stimulation is not enhanced, although there is a strong sensitization of firing in response to mechanical stimuli (Neugebauer and $\mathrm{Li}, 2003)$. These results, together with our data, suggest that plasticity in the amygdala induced by prolonged pain does not mediate thermal sensitization. An alternative explanation is that molecular pathways in the amygdala could modulate peripheral thermal hypersensitivity independent of ERK.

As mentioned above, a major projection from the CeA is to the substantia inominata dorsalis, an area critically involved in attention and memory. Therefore, one possible explanation for the effects of ERK on behavioral responses during persistent inflam- 
mation is that the CeA does not modulate pain perception, but rather acts to modulate attention. However, given that we observe effects of CeA ERK only on mechanical and not thermal hypersensitivity, we feel that this is not likely to be the case.

At the cellular level, ERK activation in the CeA could mediate plasticity that underlies the development of persistent pain. Persistent changes in neuronal excitability and synaptic transmission occur in the amygdala during chronic, but not acute pain conditions (Neugebauer et al., 2003). These plastic changes are localized to the laterocapsular division of the CeA, where we observed inflammation-induced ERK activation. Several studies have demonstrated that ERK activation mediates plasticity in various brain regions including the lateral nucleus of the amygdala, hippocampus, and in the spinal cord (Schafe et al., 2000; Sweatt, 2001; Hu and Gereau, 2003; Hu et al., 2003). The activation of ERK can lead to the activation of several transcription factors, including CREB (cAMP response element-binding protein), Elk-1, and c-Myc (Sweatt, 2001); activated ERK can also modulate neuronal excitability through posttranslational modifications of ion channels such as the voltage-gated potassium channel Kv4.2 (Sweatt, 2001; Hu et al., 2003; Hu et al., 2006). It is therefore possible that ERK activation in the CeA mediates plasticity that underlies the development of persistent pain via transcriptional and/or posttranslational modifications.

Our studies show that formalin injection induces hypersensitivity in both the injected and the noninjected hindpaw. The mechanisms underlying contralateral pain are not completely understood (Shenker et al., 2003), but it has been proposed to be mediated by sensitization of neurons in the contralateral spinal dorsal horn, possibly through intrinsic spinal cord connections (Aloisi et al., 1993). These cellular changes can be observed in decerebrate, spinalized rats, which has led to the hypothesis that the necessary and sufficient circuitry for this behavior is located at the spinal level and that contralateral pain might not involve supraspinal modulation (Fitzgerald, 1982). Although it is possible that the necessary and sufficient circuitry for contralateral pain is located at the spinal level, our results clearly demonstrate that contralateral pain in vivo has a strong supraspinal component because blockade of formalin-induced amygdala ERK activation completely eliminated hypersensitivity in the noninjected hindpaw. The modulation of contralateral pain by the amygdala could be mediated by modulation of spinal circuitry involving any of the anatomical pathways discussed above.

Finally, the present study provides a possible molecular and neurobiological explanation for the known efficacy of cognitive and behavioral approaches to pain management. Clinical studies have demonstrated a reciprocal interaction between emotionality and pain perception in chronic pain conditions (Turk, 2003). Realization of this relationship, together with the current knowledge of corticolimbic neuroplasticity and kindling, have led to the development of the "limbically augmented pain syndrome" theory (Rome and Rome, 2000). This theory states that stress and exposure to emotionally traumatic events leads to a sensitized corticolimbic state that is analogous to central sensitization in the spinal cord and brainstem, but occurs in supraspinal structures that subserve both nociceptive processing and affective regulation. Given the well established role of the amygdala in attaching affective or emotional responses to aversive environmental stimuli (LeDoux, 2003), it is possible that neuromodulation in the amygdala is involved in emotional and/or cognitive processes underlying persistent pain. Amygdala-dependent associative memories, such as Pavlovian conditioning, have been shown to have the potential to be extinguished (LeDoux, 2003). The cognitive-behavioral approach to managing chronic pain assumes that this type of extinguishable associative learning is central to maintaining chronic pain behaviors. Understanding how different molecules in the amygdala affect the perception of pain and identifying the mechanisms underlying extinction could provide insights toward the development of new therapeutic tools to be used for pain management. The extent to which amygdala ERK activation in mouse experimental models of hypersensitivity subserves a cognitive or emotional component of the pain experience and whether this ERK activation and amygdaladependent alterations in pain perception can undergo extinction are important issues that should be addressed by additional experimentation.

\section{References}

Adamec RE, Blundell J, Burton P (2003) Phosphorylated cyclic AMP response element binding protein expression induced in the periaqueductal gray by predator stress: its relationship to the stress experience, behavior and limbic neural plasticity. Prog Neuropsychopharmacol Biol Psychiatry 27:1243-1267.

Aggleton JP (2000) The amygdala: a functional analysis, Ed 2. Oxford: Oxford UP.

Aloisi AM, Porro CA, Cavazzuti M, Baraldi P, Carli G (1993) "Mirror pain” in the formalin test: behavioral and 2-deoxyglucose studies. Pain 55:267-273.

Baker KB, Kim JJ (2004) Amygdalar lateralization in fear conditioning: evidence for greater involvement of the right amygdala. Behav Neurosci 118:15-23.

Berman SM, Naliboff BD, Suyenobu B, Labus JS, Stains J, Bueller JA, Ruby K, Mayer EA (2006) Sex differences in regional brain response to aversive pelvic visceral stimuli. Am J Physiol Regul Integr Comp Physiol 291:R268-R76.

Bernard JF, Besson JM (1990) The spino(trigemino)pontoamygdaloid pathway: electrophysiological evidence for an involvement in pain processes. J Neurophysiol 63:473-490.

Bernard JF, Peschanski M, Besson JM (1989) A possible spino (trigemino)ponto-amygdaloid pathway for pain. Neurosci Lett 100:83-88.

Bernard JF, Huang GF, Besson JM (1992) Nucleus centralis of the amygdala and the globus pallidus ventralis: electrophysiological evidence for an involvement in pain processes. J Neurophysiol 68:551-569.

Bernard JF, Alden M, Besson JM (1993) The organization of the efferent projections from the pontine parabrachial area to the amygdaloid complex: a Phaseolus vulgaris leucoagglutinin (PHA-L) study in the rat. J Comp Neurol 329:201-229.

Bernard JF, Bester H, Besson JM (1996) Involvement of the spinoparabrachio -amygdaloid and -hypothalamic pathways in the autonomic and affective emotional aspects of pain. Prog Brain Res 107:243-255.

Bhave G, Karim F, Carlton SM, Gereau IV RW (2001) Peripheral group I metabotropic glutamate receptors modulate nociception in mice. Nat Neurosci 4:417-423.

Cahill L, Uncapher M, Kilpatrick L, Alkire MT, Turner J (2004) Sex-related hemispheric lateralization of amygdala function in emotionally influenced memory: an FMRI investigation. Learn Mem 11:261-266.

Coleman-Mesches K, McGaugh JL (1995) Differential involvement of the right and left amygdalae in expression of memory for aversively motivated training. Brain Res 670:75-81.

Crown ED, King TE, Meagher MW, Grau JW (2000) Shock-induced hyperalgesia: III. Role of the bed nucleus of the stria terminalis and amygdaloid nuclei. Behav Neurosci 114:561-573.

Duvarci S, Nader K, LeDoux JE (2005) Activation of extracellular signalregulated kinase- mitogen-activated protein kinase cascade in the amygdala is required for memory reconsolidation of auditory fear conditioning. Eur J Neurosci 21:283-289.

Favata MF, Horiuchi KY, Manos EJ, Daulerio AJ, Stradley DA, Feeser WS, Van Dyk DE, Pitts WJ, Earl RA, Hobbs F, Copeland RA, Magolda RL, Scherle PA, Trzaskos JM (1998) Identification of a novel inhibitor of mitogen-activated protein kinase kinase. J Biol Chem 273:18623-18632.

Fitzgerald M (1982) The contralateral input to the dorsal horn of the spinal cord in the decerebrate spinal rat. Brain Res 236:275-287.

Fox RJ, Sorenson CA (1994) Bilateral lesions of the amygdala attenuate an- 
algesia induced by diverse environmental challenges. Brain Res 648:215-221.

Gao YJ, Ren WH, Zhang YQ, Zhao ZQ (2004) Contributions of the anterior cingulate cortex and amygdala to pain- and fear-conditioned place avoidance in rats. Pain 110:343-353.

Han JS, Neugebauer V (2005) mGluR1 and mGluR5 antagonists in the amygdala inhibit different components of audible and ultrasonic vocalizations in a model of arthritic pain. Pain 113:211-222.

Han JS, Li W, Neugebauer V (2005) Critical role of calcitonin gene-related peptide 1 receptors in the amygdala in synaptic plasticity and pain behavior. J Neurosci 25:10717-10728.

Hargreaves K, Dubner R, Brown F, Flores C, Joris J (1988) A new and sensitive method for measuring thermal nociception in cutaneous hyperalgesia. Pain 32:77-88.

Helmstetter FJ, Bellgowan PS (1993) Lesions of the amygdala block conditional hypoalgesia on the tail flick test. Brain Res 612:253-257.

Helmstetter FJ, Bellgowan PS, Poore LH (1995) Microinfusion of mu but not delta or kappa opioid agonists into the basolateral amygdala results in inhibition of the tail flick reflex in pentobarbital-anesthetized rats. J Pharmacol Exp Ther 275:381-388.

Helmstetter FJ, Tershner SA, Poore LH, Bellgowan PS (1998) Antinociception following opioid stimulation of the basolateral amygdala is expressed through the periaqueductal gray and rostral ventromedial medulla. Brain Res 779:104-118.

Hu HJ, Gereau IV RW (2003) ERK integrates PKA and PKC signaling in superficial dorsal horn neurons. II. Modulation of neuronal excitability. J Neurophysiol 90:1680-1688.

Hu HJ, Glauner KS, Gereau IV RW (2003) ERK integrates PKA and PKC signaling in superficial dorsal horn neurons. I. Modulation of A-type $\mathrm{K}^{+}$ currents. J Neurophysiol 90:1671-1679.

Hu HJ, Carrasquillo Y, Karim F, Jung WE, Nerbonne JM, Schwarz TL, Gereau IV RW (2006) The kv4.2 potassium channel subunit is required for pain plasticity. Neuron 50:89-100.

Huang GF, Besson JM, Bernard JF (1993) Intravenous morphine depresses the transmission of noxious messages to the nucleus centralis of the amygdala. Eur J Pharmacol 236:449-456.

Jasmin L, Burkey AR, Card JP, Basbaum AI (1997) Transneuronal labeling of a nociceptive pathway, the spino-(trigemino-)parabrachioamygdaloid, in the rat. J Neurosci 17:3751-3765.

Jasmin L, Rabkin SD, Granato A, Boudah A, Ohara PT (2003) Analgesia and hyperalgesia from GABA-mediated modulation of the cerebral cortex. Nature 424:316-320.

Karim F, Wang CC, Gereau IV RW (2001) Metabotropic glutamate receptor subtypes 1 and 5 are activators of extracellular signal-regulated kinase signaling required for inflammatory pain in mice. J Neurosci 21:3771-3779.

Karim F, Hu HJ, Adwanikar H, Kaplan DR, Gereau IV RW (2006) Impaired inflammatory pain and thermal hyperalgesia in mice expressing neuronspecific dominant negative mitogen activated protein kinase kinase (MEK). Mol Pain 2:2.

LeDoux J (2003) The emotional brain, fear, and the amygdala. Cell Mol Neurobiol 23:727-738.

Lee GP, Meador KJ, Loring DW, Allison JD, Brown WS, Paul LK, Pillai JJ, Lavin TB (2004) Neural substrates of emotion as revealed by functional magnetic resonance imaging. Cogn Behav Neurol 17:9-17.

Lu KT, Walker DL, Davis M (2001) Mitogen-activated protein kinase cascade in the basolateral nucleus of amygdala is involved in extinction of fear-potentiated startle. J Neurosci 21:RC162(1-5).

Lu L, Hope BT, Dempsey J, Liu SY, Bossert JM, Shaham Y (2005) A critical role of central amygdala ERK signaling pathway in incubation of cocaine craving. Nat Neurosci 8:212-219.

Manning BH (1998) A lateralized deficit in morphine antinociception after unilateral inactivation of the central amygdala. J Neurosci 18:9453-9470.

Manning BH, Mayer DJ (1995a) The central nucleus of the amygdala contributes to the production of morphine antinociception in the rat tail-flick test. J Neurosci 15:8199-8213.

Manning BH, Mayer DJ (1995b) The central nucleus of the amygdala contributes to the production of morphine antinociception in the formalin test. Pain 63:141-152.

Manning BH, Merin NM, Meng ID, Amaral DG (2001) Reduction in opioid- and cannabinoid-induced antinociception in rhesus monkeys after bilateral lesions of the amygdaloid complex. J Neurosci 21:8238-8246.
Manning BH, Martin WJ, Meng ID (2003) The rodent amygdala contributes to the production of cannabinoid-induced antinociception. Neuroscience 120:1157-1170.

Mao J, Mayer DJ, Price DD (1993) Patterns of increased brain activity in dicative of pain in a rat model of peripheral mononeuropathy. J Neurosci 13:2689-2702.

Mayer EA, Berman S, Suyenobu B, Labus J, Mandelkern MA, Naliboff BD, Chang L (2005) Differences in brain responses to visceral pain between patients with irritable bowel syndrome and ulcerative colitis. Pain 115:398-409.

McGaraughty S, Heinricher MM (2002) Microinjection of morphine into various amygdaloid nuclei differentially affects nociceptive responsiveness and RVM neuronal activity. Pain 96:153-162.

Morris JS, Ohman A, Dolan RJ (1998) Conscious and unconscious emotional learning in the human amygdala. Nature 393:467-470.

Naliboff BD, Berman S, Chang L, Derbyshire SW, Suyenobu B, Vogt BA, Mandelkern M, Mayer EA (2003) Sex-related differences in IBS patients: central processing of visceral stimuli. Gastroenterology 124:1738-1747.

Neugebauer V, Li W (2002) Processing of nociceptive mechanical and thermal information in central amygdala neurons with knee-joint input. J Neurophysiol 87:103-112.

Neugebauer V, Li W (2003) Differential sensitization of amygdala neurons to afferent inputs in a model of arthritic pain. J Neurophysiol 89:716-727.

Neugebauer V, Li W, Bird GC, Bhave G, Gereau IV RW (2003) Synaptic plasticity in the amygdala in a model of arthritic pain: differential roles of metabotropic glutamate receptors 1 and 5. J Neurosci 23:52-63.

Neugebauer V, Li W, Bird GC, Han JS (2004) The amygdala and persistent pain. Neuroscientist 10:221-234.

Phelps EA, O'Connor KJ, Gatenby JC, Gore JC, Grillon C, Davis M (2001) Activation of the left amygdala to a cognitive representation of fear. Nat Neurosci 4:437-441.

Roberson ED, English JD, Adams JP, Selcher JC, Kondratick C, Sweatt JD (1999) The mitogen-activated protein kinase cascade couples PKA and $\mathrm{PKC}$ to cAMP response element-binding protein phosphorylation in area CA1 of hippocampus. J Neurosci 19:4337-4348.

Rome Jr HP, Rome JD (2000) Limbically augmented pain syndrome (LAPS): kindling, corticolimbic sensitization, and the convergence of affective and sensory symptoms in chronic pain disorders. Pain Med $1: 7-23$.

Schafe GE, Atkins CM, Swank MW, Bauer EP, Sweatt JD, LeDoux JE (2000) Activation of ERK/MAP kinase in the amygdala is required for memory consolidation of pavlovian fear conditioning. J Neurosci 20:8177-8187.

Schneider F, Habel U, Holthusen H, Kessler C, Posse S, Muller-Gartner HW, Arndt JO (2001) Subjective ratings of pain correlate with subcorticallimbic blood flow: an fMRI study. Neuropsychobiology 43:175-185.

Shane R, Lazar DA, Rossi GC, Pasternak GW, Bodnar RJ (2001) Analgesia elicited by $\mathrm{OFQ} /$ nociceptin and its fragments from the amygdala in rats. Brain Res 907:109-116.

Shane R, Acosta J, Rossi GC, Bodnar RJ (2003) Reciprocal interactions between the amygdala and ventrolateral periaqueductal gray in mediating of Q/N(1-17)-induced analgesia in the rat. Brain Res 980:57-70.

Shenker N, Haigh R, Roberts E, Mapp P, Harris N, Blake D (2003) A review of contralateral responses to a unilateral inflammatory lesion. Rheumatology (Oxford) 42:1279-1286.

Sweatt JD (2001) The neuronal MAP kinase cascade: a biochemical signal integration system subserving synaptic plasticity and memory. J Neurochem 76:1-10.

Thiels E, Klann E (2001) Extracellular signal-regulated kinase, synaptic plasticity, and memory. Rev Neurosci 12:327-345.

Tjolsen A, Berge OG, Hunskaar S, Rosland JH, Hole K (1992) The formalin test: an evaluation of the method. Pain 51:5-17.

Turk DC (2003) Cognitive-behavioral approach to the treatment of chronic pain patients. Reg Anesth Pain Med 28:573-579.

Yang D, Gereau IV RW (2003) Peripheral group II metabotropic glutamate receptors mediate endogenous anti-allodynia in inflammation. Pain 106:411-417.

Yuan LL, Adams JP, Swank M, Sweatt JD, Johnston D (2002) Protein kinase modulation of dendritic $\mathrm{K}^{+}$channels in hippocampus involves a mitogen-activated protein kinase pathway. J Neurosci 22:4860-4868. 